\title{
A Novel Hand Prosthesis Control Scheme Implementing a Tongue Control System ${ }^{1}$
}

\author{
Daniel Johansen ${ }^{\mathrm{a}}$, Dejan B. Popović ${ }^{\mathrm{b}}$, Lotte N.S.A. Struijk ${ }^{\mathrm{c}}$, Fredrik Sebelius ${ }^{\mathrm{d}}$, Stig Jensen ${ }^{\mathrm{e}}$ \\ ${ }^{a, b, c}$ Department of Health Science and Technology, Aalborg University, Aalborg, Denmark \\ ${ }^{d}$ Department of Electrical Measurements, Lund University, Lund, Sweden \\ ${ }^{e}$ Arm Center, Sahva A/S, Copenhagen, Denmark
}

\begin{abstract}
This paper presents a novel control scheme for new advanced hand prostheses implementing multiple pinches and grasps. The control signals for the hand are determined by myoelectric signals from the arm and volitionally generated signals by tongue through an inductive interface with a mouth piece. The feasibility tests have been performed on an able-bodied subject who controller a virtual reality hand. Results suggest that this hybrid control scheme works well, it is intuitive and the subject learned fast to use it, and what is most important provides many more control channel to advanced hand prostheses. This research is being continued in healthy subjects with the intention to translate the results to patients.
\end{abstract}

Index Terms: Component; Hand Prosthesis; Prosthesis Control System; Inductive Tongue Control System

(C) 2012 Published by MECS Publisher. Selection and/or peer review under responsibility of the International Conference on E-Business System and Education Technology

\section{Introduction}

Most commercially available electrical hand prostheses solely implement the three-finger precision pinch, involving the thumb, index and middle finger of the hand prosthesis. An example of such a prosthetic device is the SensorHand Speed ${ }^{\circledR}$ from Otto Bock $®$, Germany.

The precision pinch covers only $20 \%$ of the Activities of Daily Living (ADL) [1]. This limited functionality, in the context of ADL, has also been confirmed through questionnaires and surveys [2,3]. Aside from the desire to have lighter, more reliable and more anthropomorphic prostheses, it is found that a general demand among hand and arm prostheses users' is improved digit movement and grasp functions [2, 3].

These demands are also reflected in the research and development of hand prostheses. New advanced prostheses are implementing several grasps and greater dexterity, and thus potentially allowing the amputee a

\footnotetext{
${ }^{1}$ This work was supported in part by Bevica Innovation A/S, The Danish Agency for Science, Technology and Innovation and Sahva A/S. Dejan Popović is also with the University of Belgrade, School of Electrical Engineering, Begrlade, Serbia

Corresponding author:

E-mail address: ${ }^{a}$ djoh@hst.aau.dk
} 
superior use of the prosthetic device. Examples of such prostheses are the Vincenthand, available from medical technics by Vincent Systems GmbH; the i-Limb, commercially available from Touch Bionics SmartHand, which will be commercially available from Prensilia S.R.L. and the DEKA arm, from the revolutionizing prosthetics program. These hand prostheses implement grasps, which cover up to 79\% of ADL [1].

With these additional grasps and functions follows the demand for new control schemes that allow an easy and intuitive control of the prosthetic arm and/or hand. Recent research on control signals and control schemes for hand and arm prostheses includes MyoElectric Signals (MES) as well as alternative control signals.

Regarding MES recorded from the arm or forearm of the amputee, pattern recognition algorithms are being used in order to extract more information from the remaining muscles [4, 5]. However the usability of control schemes based on surface MES recorded from the arm or forearm of the amputee are highly influenced by the level of amputation. For high-level amputees, the number of available muscles decreases whereas the amount of functionality to be recovered through the prosthesis increases. Therefore the use of other types of control signals, e.g. foot switches, shoulder joysticks and throat microphones [6-8], are being investigated.

Recently, the combination of an artificial vision for detection of the prehension and hand orientation has been tested in combination with the myoleectric trigger control from the forearm with one of the versions of SmartHand [9]. The tests suggested that adding another input to the myoelectric control is an important element in benefiting form the advanced multiactuated hand prostheses.

This paper presents a novel control scheme for hand prostheses in which control signals from an inductive tongue control system [10] are combined with standard surface MES, recorded from the arm or forearm of the amputee, to obtain an efficient control of multiple grasping modalities.

\section{Methods}

\subsection{The Inductive Tongue Control System}

The Inductive Tongue Control System (ITCS) is a fully integrated wireless inductive tongue interface, incorporating 18 separate inductive sensors [11]. The system consists of a mouthpiece unit, an activation unit and a central unit. The mouthpiece unit is placed in the upper palatal area, similar to a dental brace. The activation unit is fixed to the tip of the tongue using bio-compatible tissue-glue, and when placed on a sensor, the sensor is activated. The onboard electronics of the mouthpiece unit scan the 18 inductive sensors of the mouthpiece every $33 \mathrm{~ms}$. The sensor output is then amplified, rectified, low pass filtered and sent wirelessly to the central unit of the ITCS, which processes the signals values to obtain a decision on which sensor is activated [11]. Then, the central unit transmits the processed signal to the desired interface serial port or radio receiver (e.g. wheelchairs control BUS, computers USB-radio receiver, or prosthetics controller).

\subsection{The ITCS Control Scheme}

The ITCS control scheme for hand prostheses provides the amputee with the possibility to select and activate desired functions of the prosthetic device using the ITCS (Fig. 1). Opening and closing of a selected pinch or grasp or operating a function, e.g. wrist rotation, is then done using MES signals recorded from the arm or forearm. The electrodes used for recording of the MES need to be connected to the central unit, and the central unit connected to the controller of the hand prosthesis. For the proposed use in prostheses control schemes the ITCS's central unit could be incorporated in the prosthesis socket. 




Fig. 1. The tongue control system allows the user to directly select between or activate a desired function or grasp of hand prosthesis. Up to 18 functions or grasps (dashed) could be implemented in the ITCS control scheme

\subsection{Experimental Setup}

Based on a prototype of the SmartHand prosthesis, a central unit implementing a ITCS control scheme is developed. The control scheme implements the use of five pinches and grasps; precision pinch, lateral pinch, diagonal volar grasp, transversal volar grasp and a tripod pinch (Fig. 2). Table 1 shows the representation of each of these five pinches and grasps in ADL. Using the individual representation of a pinch or grasp and the accessibility of the ITCS sensors (Fig. 3A) [12] each of the five pinches and grasps are allocated to sensors in the mouthpiece unit. Ten of the 18 sensors available are used in the proposed ITCS control scheme. The ten sensors are paired (Fig. 3B) and the most used pinch or grasp is allocated to the sensor pair that has the highest combined accessibility.
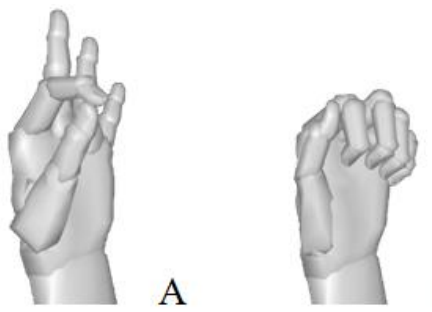

B
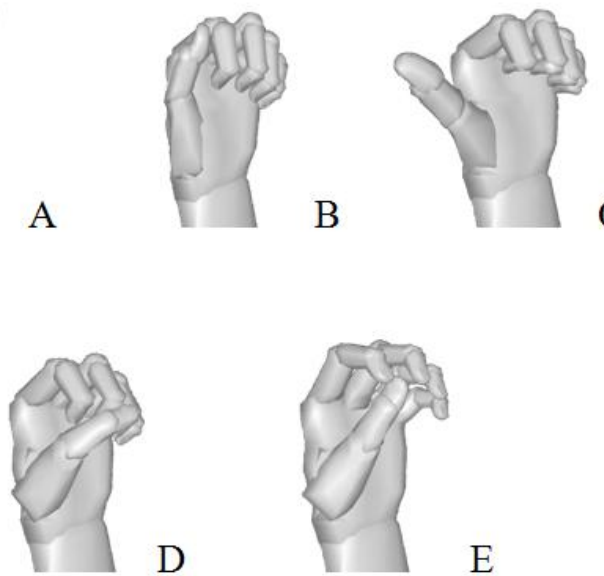

Fig. 2. The five implemented grasp patterns of the hand prosthesis computer model:

A: Precision grasp, B: Lateral grasp, C: Transversal volar grasp, D: Diagonal volar grasp, E: Three finger grasp 
Table 1. The five pinches and grasps that is implemented in the ITCS control scheme, and their representation in activities of daily living according to [1]

\begin{tabular}{lc}
\hline \multicolumn{1}{c}{ Representations of Pinches and Grasp in Activities of Daily Living } \\
\hline \multicolumn{1}{c}{ Pinch or Grasp } & Representation in Activities of Daily Living \\
\hline 1 Precision Pinch & $20 \%$ \\
2 Lateral Pinch & $20 \%$ \\
3 Diagonal Volar Grasp & $15 \%$ \\
4 Transversal Volar Grasp & $14 \%$ \\
5 Tripod Pinch & $10 \%$ \\
\hline
\end{tabular}

Fig. 3. A: The accessibility of the ITCS sensors measured as correct activations per second (CAPS) [11].

B: The layout of the five pinches and grasps of the ITCS control scheme; 1: precision pinch, 2: lateral pinch, 3: diagonal volar grasp, 4: transversal volar grasp, 5: tripod pinch


For recording of MES two 13E200 MYOBOCK electrodes, from Otto Bock®, are connected to the central unit. The output of the 13E200 is an amplified, filtered, rectified and enveloped representation of the MES. Both electrode output signals are sampled each time the central unit receives a transmission from the mouthpiece unit.

In order to test the ITCS control scheme, a computer model resembling the movements of an actual SmartHand prosthesis is used. The computer model is based on the VirtualHand software from Virtual Technologies Inc., USA, which is an 18 degree of freedom computer model of the human hand. The original VirtualHand software is modified to allow the use of the five implemented pinches and grasps. When a pinch or grasp is selected, the computer model assumes the unique preshaped position of that pinch or grasp, which can then be closed or opened either fully or partially.

The computer model can be controlled using standard keyboard commands. Therefore a wireless USB keyboard emulator receives signals from the central unit and generates the corresponding keyboard event. The different preshapes of the computer model can then be activated using the ITCS and the closing and opening of pinches and grasps is done using the recorded MES signals. 
The mouthpiece unit used in the test of the ITCS control scheme is a prototype. This prototype mouthpiece unit solely holds the inductive sensor boards inside the mouth. The electronic circuits of the mouthpiece unit are placed externally, connected to the sensor boards via copper cables insulated by a silicone tube. If the prototype mouthpiece is placed in the mouth, the silicone tube exits at the corner of the mouth.

\subsection{Subject}

A 26 year old healthy female, with no prior training with the ITCS was used for demonstrating the functionality of the proposed ITCS control scheme for hand prostheses.

\subsection{Experimental Protocol}

The protocol used in this test was approved by the local ethics committee. The subject trained for three consecutive days. Each training session consisted of 25 exercises. An exercise was completed by correctly activating a pinch or grasp, completely closing it and then completely opening it again. Each session included six precision pinch and lateral pinch exercises, five diagonal volar grasp and the transversal volar grasp exercises and three tripod pinch exercises. The order of exercises was randomized for each training session. The number of times that each exercise was included in the training sessions was based on the representation of the involved pinch or grasp in ADL [1]. This was done in order to ensure that the subject is trained in a way that resembles the normal use of a prosthesis, which was needed for a planned ensuing study.

At the start of each training session the recording electrodes were placed on the left forearm. The electrode for closing pinches and grasps was placed on the anterior side and the electrode for opening was placed on posterior side. Then the activation unit was glued to the tip of the tongue of the subject using tissue glue Histoacryl®, and the mouthpiece was fixed inside the subject's mouth.

For each of the 25 exercises in a training session the times needed to select the correct preshape, and the total time needed to complete the exercise was recorded. The strategy for activating a specific grasp or pinch used by subjects, was to activate the precision pinch located at the most accessible part of the upper palatal area, and then slide or move the activation unit towards the desired pinch or grasp. Thus if subjects activated a pinch or grasp before activating the desired pinch or grasp, this would not decrement the accuracy but only increment the activation time. be comparable to the reported accuracy of e.g. a pattern recognition algorithm. The accuracy parameters normally reported in studies on prosthesis control schemes are therefore not reported.

\section{Results}

Both the mean time for activating a desired pinch or grasp (Fig. 4) and the mean time used to complete the exercises (Fig. 5) decreased between training session one and two. And both the mean time used for activation as well as for completion of exercises increased between sessions two and three, with the exception of the tripod pinch. 




Fig. 4. Mean values for the total time used to activate a specific preshape of the computer model using an ITCS control scheme

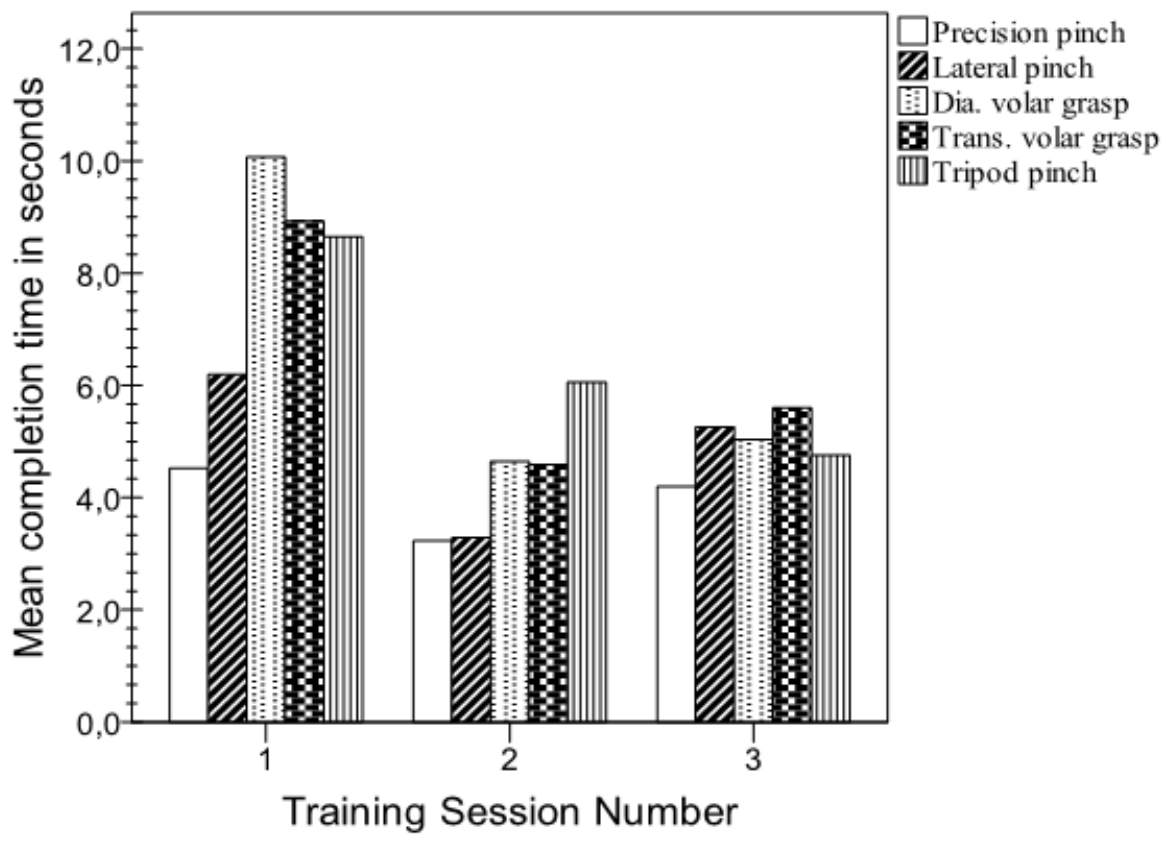

Fig. 5. Mean values for the total time used to complete the pinch and grasp exercises on the computer model using an ITCS control scheme 
After three training sessions of approximately 20 minutes the mean time used to activate one of the five pinches or grasps was 1.4 seconds, and the mean time used to complete the exercises was 4.7 seconds.

\section{Conclusion}

A novel control scheme for hand prostheses is proposed. The control scheme combines myoelectric signals with a novel inductive tongue control system.

The proposed control scheme has been tested using a computer model that implements the pinches and grasps of a SmartHand prosthesis prototype.

Based on the amount of training and the achieved level of performance by the subject used in the test, it is concluded that the proposed control scheme could prove to be an easy and intuitive method of enhancing prosthesis control. Further studies are needed to establish this assumption. This should be done by recruiting more subjects and preferably implementing the control scheme on an actual hand prosthesis instead of a computer model.

\section{Acknowledgements}

Henrik Vie Christensen, PhD. is acknowledged for his help with hardware and software design and debugging. Bo Bentsen, cand.odont., $\mathrm{PhD}$, is acknowledged for being clinical responsible for the experimental protocol.

\section{References}

[1] C. Sollerman and A. Ejeskar, "Sollerman hand function test. A standardised method and its use in tetraplegic patients," Scand. J. Plast. Reconstr. Surg. Hand Surg., vol. 29, pp. 167-176, Jun. 1995.

[2] P. J. Kyberd, C. Wartenberg, L. Sandsjö, S. Jönsson, D. Gow, J. Frid, C. Almström and L. Sperling, "Survey of Upper-Extremity Prosthesis Users in Sweden and the United Kingdom," JPO Journal of Prosthetics and Orthotics, vol. 19, pp. 55, 2007.

[3] C. Pylatiuk, S. Schulz and L. Doderlein, "Results of an Internet survey of myoelectric prosthetic hand users," Prosthet. Orthot. Int., vol. 31, pp. 362-370, Dec. 2007.

[4] Li Guanglin, A.E Schultz, T.A. Kuiken, "Quantifying Pattern Recognition-Based Myoelectric Control of Multifunctional Transradial Prostheses," Neural Systems and Rehabilitation Engineering, IEEE Transactions on , vol.18, no.2, pp.185-192, 2010

[5] L. Hargrove, E. Scheme, K. Englehart, B. Hudgins, "Multiple Binary Classifications via Linear Discriminant Analysis for Improved Controllability of a Powered Prosthesis," Neural Systems and Rehabilitation Engineering, IEEE Transactions on , vol.18, no.1, pp.49-57, 2010

[6] M. C. Carrozza, A. Persichetti, C. Laschi, F. Vecchi, R. Lazzarini, V. Tamburrelli, P. Vacalebri and P. Dario, "A novel wearable interface for robotic hand prostheses," in Proceedings of the 2005 IEEE 9th International Conference on Rehabilitation Robotics, 2005, pp. 109-112.

[7] Y. Losier, K. Englehart and B. Hudgins, "A control system for a powered prosthesis using positional and myoelectric inputs from the shoulder complex," Conf. Proc. IEEE Eng. Med. Biol. Soc., vol. 2007, pp. 6138-6141, 2007.

[8] E. Mainardi and A. Davalli, "Controlling a prosthetic arm with a throat microphone," Conf. Proc. IEEE Eng. Med. Biol. Soc., vol. 2007, pp. 3035-3039, 2007. 
[9] S. Došen, C. Cipriani, M. Kostić, M. C. Carrozza, D. B. Popović, „Cognitive vision system for the control of a dexterous prosthetic hand: An evaluation study.” J NeuroEng Rehabil 2010, 7:42 doi:10.1186/17430003-7-42

[10] L. N. Struijk, "An inductive tongue computer interface for control of computers and assistive devices," IEEE Trans. Biomed. Eng., vol. 53, pp. 2594-2597, Dec. 2006.

[11] L. N. Struijk, E. R. Lontis, B. Bentsen, H. V. Christensen, H. A. Caltenco and M. E. Lund, "Fully integrated wireless inductive tongue computer interface for disabled people," Conf. Proc. IEEE Eng. Med. Biol. Soc., vol. 2009, pp. 547-550, 2009.

[12] H. A. Caltenco, E. R. Lontis, S. Boudreau, B. Bentsen, L. N. Struijk "Tip of the tongue selectivity and motor learning around the palatal area" unpublished. 